\title{
Surgery provides improved overall survival in surgically fit octogenarians with esophageal cancer after chemoradiation therapy
}

\author{
Haydee Del Calvo ${ }^{1}$, Duc T. Nguyen ${ }^{2}$, Edward Y. Chan ${ }^{1,3}$, Ray Chihara ${ }^{1,3}$, Edward A. Graviss ${ }^{2}$, Min P. Kim ${ }^{1,3}$ \\ ${ }^{1}$ Division of Thoracic Surgery, Department of Surgery, Houston Methodist Hospital, Houston, TX, USA; ${ }^{2}$ Department of Pathology and Genomic \\ Medicine, Houston Methodist Research Institute, Houston, TX, USA; ${ }^{3}$ Department of Surgery and Cardiothoracic Surgery, Weill Cornell Medical \\ College, Houston Methodist Hospital, Houston, TX, USA \\ Contributions: (I) Conception and design: MP Kim, EY Chan, R Chihara, EA Graviss, H Del Calvo; (II) Administrative support: EA Graviss, MP \\ Kim; (III) Provision of study materials of patients: EA Graviss, MP Kim; (IV) Collection and assembly of data: EA Graviss, DT Nguyen; (V) \\ Data analysis and interpretation: EA Graviss, DT Nguyen, H Del Calvo, MP Kim; (VI) Manuscript writing: All authors; (VII) Final approval of \\ manuscript: All authors. \\ Correspondence to: Min P. Kim, MD, FACS. 6550 Fannin Street, Suite 1661, Houston, TX 77030, USA. Email: mpkim@houstonmethodist.org.
}

Background: Multiple randomized controlled trials have shown that multimodal therapy provides the best overall survival for patients who had locally advanced esophageal cancer. However, it is unknown if multimodal therapy offers the best overall survival in octogenarians.

Methods: We performed retrospective cohort study using data obtained from the National Cancer Database (NCDB) for octogenarians who had locally advanced esophageal cancer from 2004 to 2015. We evaluated the 5-year overall survival for patients among different therapies. We compared the 5 -year overall survival between patients receiving chemoradiation therapy followed by surgery and a propensity-matched group of patients who underwent chemoradiation only.

Results: There were 21,710 octogenarians (15\%) with esophageal cancer in the NCDB database. Among octogenarians, there were 6,960 patients (32\%) who had clinical stage II-III esophageal cancer. Among 6,922 patients whose treatment data were available, the most common therapy was chemoradiation $(n=3,360$, $49 \%)$. Two of the most common therapies that included surgical resection were surgery only $(\mathrm{n}=314,5 \%)$ and chemoradiation therapy followed by surgery (n=172, 2\%). Among different treatments, the best 5 -year overall survival was achieved in patients receiving chemoradiation therapy followed by surgery $(\mathrm{P}<0.001)$. In the propensity score-matched cohort between chemoradiation therapy followed by surgery $(n=83)$ to chemoradiation therapy only $(n=83)$, there was an association with improved 5 -year overall survival in the patients who had chemoradiation therapy followed by surgery (17.9\%) compared to the patients who underwent chemoradiation only $(5.7 \%, \mathrm{P}=0.003)$.

Conclusions: Most octogenarians with locally advanced esophageal cancer underwent definitive chemoradiation therapy. Very few patients underwent chemoradiation followed by surgery; however, the multimodality treatment provided increased overall survival. Surgically fit octogenarians should be considered for chemoradiation therapy followed by surgery.

Keywords: Esophageal cancer; octogenarians; esophagectomy; chemoradiation therapy

Submitted Jun 04, 2021. Accepted for publication Aug 19, 2021.

doi: $10.21037 /$ jtd-21-928

View this article at: https://dx.doi.org/10.21037/jtd-21-928 


\section{Introduction}

According to the National Institute of Cancer, the incidence of esophageal cancer was 4.2 per 100,000 men and women per year, with the 5 -year overall survival only about $20 \%$ (1). Of those patients diagnosed with esophageal cancer, about $50 \%$ were diagnosed with either tumor localized to the primary site or regionally advanced to nearby lymph nodes (2). Unfortunately, the 5 -year overall survival for localized tumors is at best $40 \%$, but only if there is no evidence of regional spread (1). Fortunately, research continues to increase understanding and test the boundaries of perfecting treatment modalities. The current standard of care for locoregional cancer, stage II and III, includes neoadjuvant chemotherapy or chemoradiation therapy followed by surgical resection (3-5). However, it is unclear if this standard of care should be applied to octogenarians. Studies that established induction chemotherapy or chemoradiation therapy followed by surgery as the standard of care did not concentrate on patients greater than 80 years old $(3,4)$. Thus, it is unclear if multimodal treatment should be applied to this cohort of patients.

In 2018, a population-based study of the National Institute of Cancer database analyzed data of 61,799 patients with esophageal cancer and divided the cohort into two age groups: patients less than 70 years of age and patients 70 years of age or older at diagnosis. Elderly patients were found to undergo surgery less often and had a higher rate of not undergoing any therapy. Interestingly, this was not related to being diagnosed at a more advanced stage since the elderly group showed a higher proportion of localized disease at diagnosis. On the other hand, overall survival was lower in the patient group greater than 70 years of age. A multivariate analysis of their data showed that the risk of mortality was increased in the elderly and that treatment modality of surgery and/or radiation therapy was an independent favorable prognostic factor (6). As further studies pursue subset analysis with a higher age threshold to define the elderly, such as the age of 80 , they continue to show even more significant associations between mortality and elderly age. For instance, a systematic review and pooled analysis of esophagectomy outcomes in octogenarians looked at a total of 2,573 patients and found octogenarians to have reduced overall and 5-year cancerfree survival (7). At the same time, there have been multiple smaller scale studies that show that while overall and cancer-specific survival is decreased with increasing age, the perioperative morbidity and mortality are not statistically different when compared to patients $<70$ years of age. Interestingly, this study also references that unwillingness to continue multimodal therapy post-operatively can be a reason for decreased cancer specific survival $(8,9)$. Given such varied data and no consensus on a treatment course for the elderly population, we aimed to determine if conducting the multimodality treatment in octogenarians with locally advanced esophageal cancer would provide a survival benefit.

We present the following article in accordance with the STROBE reporting checklist (available at https://dx.doi. org/10.21037/jtd-21-928).

\section{Methods}

The study was conducted in accordance with the Declaration of Helsinki (as revised in 2013). The institutional review board approval was waived for this study since we performed an analysis of national de-identified patient data and individual consent for this retrospective analysis was waived. We performed a retrospective cohort study using data obtained from the National Cancer Database (NCDB) on patients who were diagnosed with esophageal cancer 20042015. We excluded patients under the age of 80 , patients without a TNM stage of II or III, patients with unknown treatment, and patients with missing vital data. We analyzed baseline characteristics including age at diagnosis, sex, race, ethnicity, insurance type, median income, geographic setting, Charlson-Deyo Score, primary tumor site, tumor histology, tumor size, American Joint Committee on Cancer (AJCC) pathologic stage, lymphovascular invasion, time from diagnosis to treatment, time from diagnosis to death or last contact, and treatment facility type. Patients were divided into seven different treatment groups, including no therapy, surgery only, radiation only, chemoradiation only, chemoradiation followed by surgery, chemotherapy only, and surgery followed by chemoradiation.

\section{Statistical analysis}

Baseline characteristics were reported as frequencies and proportions for categorical variables and median and interquartile range (IQR) for continuous variables. Differences between groups were determined by the Chisquare or Fisher's exact tests for categorical variables and Kruskal Wallis test for continuous variables as appropriate. Cox proportional hazard modeling was conducted to determine the patient characteristics and treatment group 
associated with higher mortality. Patient's overall survival was depicted by the Kaplan-Meier curves and stratified by treatment group. Differences between groups were compared by the log-rank test. Sub-analyses for patient overall survival with Kaplan Meier curves were conducted in propensity score-matched patients undergoing chemoradiation only $v s$. chemoradiation followed by surgery. Nearest propensity score matching (without replacement, ratio 1:1, and caliper of 1) between patients undergoing chemoradiation only and patients having chemoradiation followed by surgery was conducted based on age, sex, race, ethnicity, Charlson-Deyo score, histology (adenocarcinoma versus not adenocarcinoma), and the AJCC clinical stage. The balance of covariates in the matched cohort was evaluated based on the standardized mean differences (10). The performance of the models was determined by the C-statistic. All the analyses were performed on Stata version 17.0 (StataCorp LLC, College Station, TX, USA). A P value of $<0.05$ was considered statistically significant.

\section{Results}

The NCDB had a total of 141,490 patients diagnosed with esophageal cancer between 2004 and 2015. In 21,710 (15\%) octogenarians, 6,922 met our inclusion and exclusion criteria and were included in the analysis (Figure 1). The median age at diagnosis for the entire cohort of 6,922 patients was 83 years, with most patients were male (68\%), white $(91 \%)$, non-Hispanic (91\%) with a Charlson-Deyo score of $0(71 \%)$. Most tumors were adenocarcinoma $(4,261 / 6,922,62 \%)$ while most of the non-adenocarcinomas were squamous cell carcinoma $(2,387 / 2,661,90 \%)$, and the median tumor size was $40 \mathrm{~mm}$, with most tumors located in the distal third of the esophagus (58\%). Of this octogenarian group of 6,922 patients with the above-mentioned characteristics, there were the following treatment groups: no therapy (19\%), surgery only (5\%), radiation only (20\%), chemoradiation only (49\%), chemoradiation followed by surgery $(2 \%)$, chemotherapy only $(4 \%)$, and surgery followed by chemoradiation (2\%) (Table 1, Figure 2).

Kaplan Meier survival analysis stratified by treatment group indicated that the overall survival was poorer in patients having no therapy and improved in treatment groups that included surgery. Among these groups, the most improved 5 -year overall survival was achieved with chemoradiation therapy followed by surgery $(\mathrm{P}<0.001$, Figure 3). The multivariable Cox proportional hazard model for mortality showed significant better overall survival in patients received any treatment compared to patients having no therapy $(\mathrm{P}<0.001)$. Better overall survival was also observed in patients being treated at an academic/research program (HR 0.84, 95\% CI: 0.76, 0.92, $\mathrm{P}<0.001$ ), and having an adenocarcinoma histologic diagnosis (HR 0.91, 95\% CI: 0.86, 0.98, $\mathrm{P}<0.001)$. On the other hand, higher mortality was found in patients of older age at diagnosis (HR 1.03, 95\% CI: 1.02, 1.04, $\mathrm{P}<0.001$ ), male sex (HR 1.07, 95\% CI: 1.01, 1.14, $\mathrm{P}=0.02$ ), having Charlson-Deyo score $>0$ (Charlson-Deyo score $=1$, HR 1.22, 95\% CI: 1.14, 1.29, $\mathrm{P}<0.001$ and Charlson-Deyo score $=2$, HR 1.42, 95\% CI: $1.27,1.58, \mathrm{P}<0.001)$, and having an increased AJCC clinical stage (HR 1.39, 95\% CI: 1.32, 1.46, $\mathrm{P}<0.001$, Table 2).

In order to control for confounding factors affecting the overall survival, we performed a propensity score matching between a subgroup of patients who received the chemoradiation followed by surgery versus patients who only received the chemoradiation. There were 166 patients were obtained from the propensity score match, with 83 patients in each group. There was no significant difference between the age at diagnosis, sex, race, ethnicity, primary payor, median income, geographic setting, Charlson-Deyo score, primary tumor site, histologic diagnosis, size of the tumor, AJCC clinical-stage, lymphovascular invasion, and facility type (Table 3). The Kaplan Meier survival curve for this matched group comparing chemoradiation followed by surgery and chemoradiation alone showed a statistically improved overall survival for the chemoradiation followed by the surgery treatment group $(18 \%$ vs. $6 \%, \mathrm{P}=0.003$, Figure 4). The multivariable Cox proportional hazard model for mortality showed a significant benefit of undergoing chemoradiation therapy followed by surgery compared to chemoradiation alone (HR 0.49, 95\% CI: 0.32, 0.74, $\mathrm{P}=0.02)$ and marginal benefit of undergoing treatment at East South Central (HR 0.19, 95\% CI: 0.03, 1.00, P=0.049). On the other hand, there was significantly poorer survival with increased age at the time of diagnosis (HR 1.13, 95\% CI: 1.02, 126, $\mathrm{P}=0.02$, Table 4).

\section{Conclusions}

Previous analyses using data obtained from the national surveillance, epidemiology, and end results (SEER) database (6), the NCDB (11), and pooled analyses of a series of esophageal cancer patients undergoing surgery $(7,12)$ showed that increasing age is a major risk factor that is associated with a poorer overall survival. A systematic 


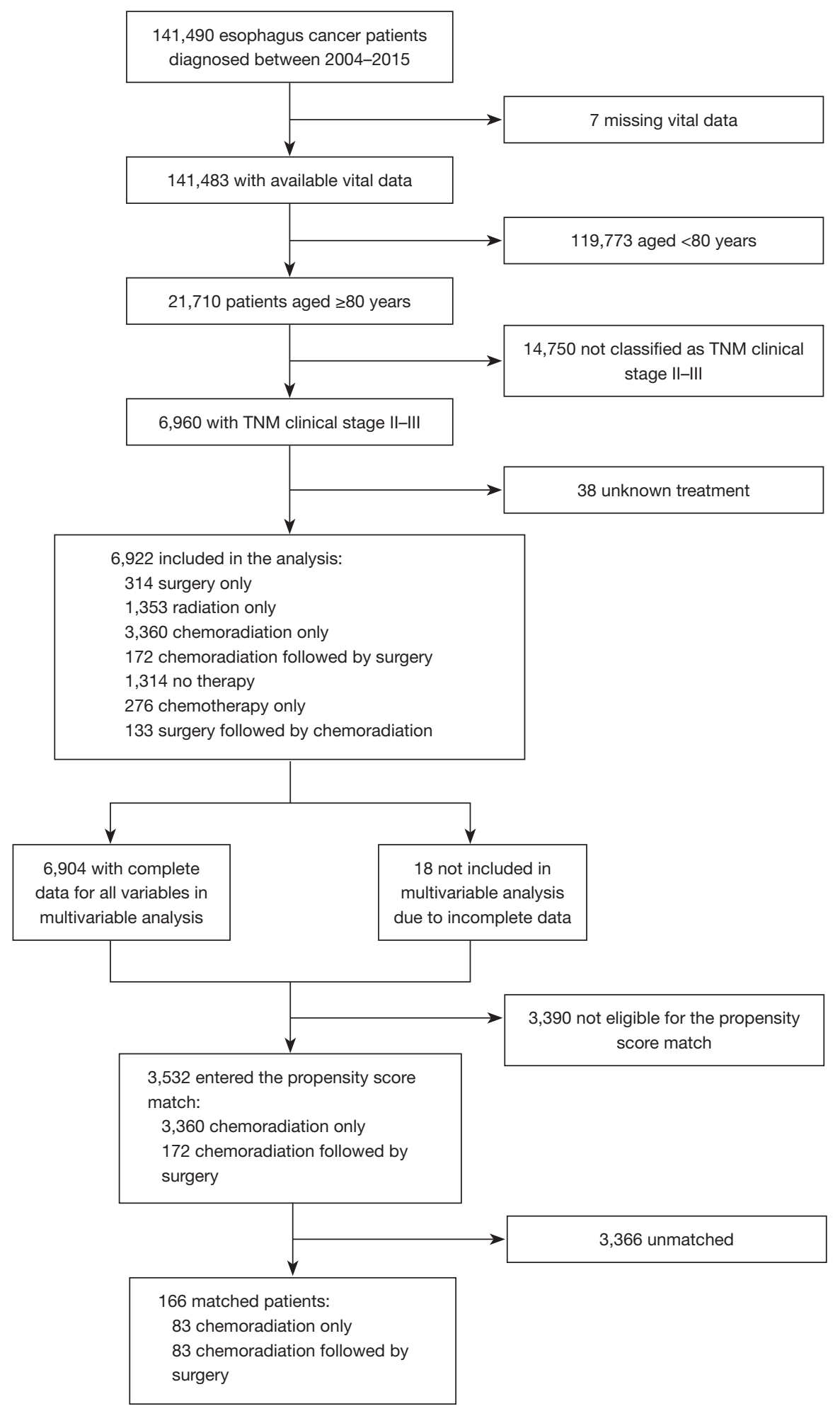

Figure 1 Flowchart of study population. One hundred forty-one thousand four hundred ninety patients in the National Cancer Database were diagnosed with esophageal cancer. Six thousand nine hundred sixty patients met the inclusion and exclusion criteria for the study. One hundred sixty-six patients were then matched for the propensity score analysis. 
Table 1 Baseline patient characteristics

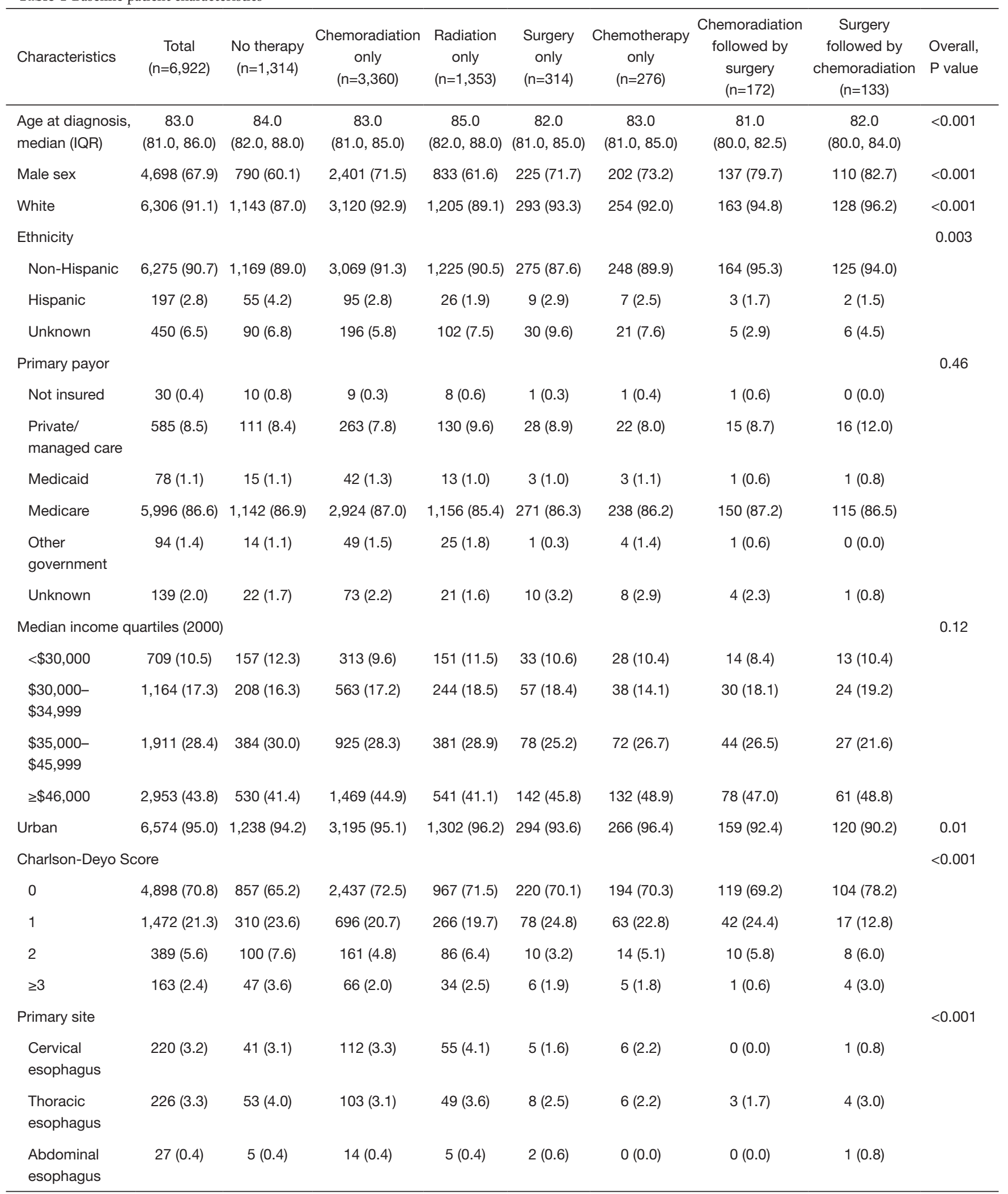

Table 1 (continued) 
Table 1 (continued)

\begin{tabular}{|c|c|c|c|c|c|c|c|c|c|}
\hline Characteristics & $\begin{array}{c}\text { Total } \\
(\mathrm{n}=6,922)\end{array}$ & $\begin{array}{l}\text { No therapy } \\
(n=1,314)\end{array}$ & $\begin{array}{c}\text { Chemoradiation } \\
\text { only } \\
(n=3,360)\end{array}$ & $\begin{array}{c}\text { Radiation } \\
\text { only } \\
(n=1,353)\end{array}$ & $\begin{array}{c}\text { Surgery } \\
\text { only } \\
(n=314)\end{array}$ & $\begin{array}{c}\text { Chemotherapy } \\
\text { only } \\
(n=276)\end{array}$ & $\begin{array}{c}\text { Chemoradiation } \\
\text { followed by } \\
\text { surgery } \\
(n=172)\end{array}$ & $\begin{array}{c}\text { Surgery } \\
\text { followed by } \\
\text { chemoradiation } \\
\quad(n=133)\end{array}$ & $\begin{array}{l}\text { Overall, } \\
\text { P value }\end{array}$ \\
\hline Upper third & $453(6.5)$ & $111(8.4)$ & $201(6.0)$ & $116(8.6)$ & $8(2.5)$ & $10(3.6)$ & $6(3.5)$ & $1(0.8)$ & \\
\hline Middle third & $1,059(15.3)$ & $222(16.9)$ & $505(15.0)$ & $220(16.3)$ & $37(11.8)$ & $42(15.2)$ & $18(10.5)$ & $15(11.3)$ & \\
\hline $\begin{array}{l}\text { Esophagus, } \\
\text { NOS }\end{array}$ & $590(8.5)$ & $168(12.8)$ & $222(6.6)$ & $134(9.9)$ & $26(8.3)$ & $28(10.1)$ & $3(1.7)$ & $9(6.8)$ & \\
\hline Adenocarcinoma & $4,261(61.6)$ & $716(54.5)$ & $2,137(63.6)$ & $731(54.0)$ & $231(73.6)$ & $190(68.8)$ & $141(82.0)$ & $115(86.5)$ & $<0.001$ \\
\hline Stage 2 & 3,685 (53.2) & $609(46.3)$ & $1,761(52.4)$ & $798(59.0)$ & 229 (72.9) & $115(41.7)$ & $88(51.2)$ & $85(63.9)$ & \\
\hline Stage 3 & $3,237(46.8)$ & $705(53.7)$ & $1,599(47.6)$ & $555(41.0)$ & $85(27.1)$ & $161(58.3)$ & $84(48.8)$ & $48(36.1)$ & \\
\hline Facility type & & & & & & & & & $<0.001$ \\
\hline $\begin{array}{l}\text { Community } \\
\text { cancer program }\end{array}$ & 735 (10.6) & $139(10.6)$ & $378(11.3)$ & $154(11.4)$ & $9(2.9)$ & $28(10.1)$ & $8(4.7)$ & 19 (14.3) & \\
\hline $\begin{array}{l}\text { Comprehensive } \\
\text { community } \\
\text { cancer program }\end{array}$ & $2,949(42.6)$ & $548(41.7)$ & $1,506(44.8)$ & $626(46.3)$ & $83(26.4)$ & $93(33.7)$ & $44(25.6)$ & $49(36.8)$ & \\
\hline New England & $662(9.6)$ & $129(9.8)$ & $323(9.6)$ & $152(11.2)$ & $17(5.4)$ & $12(4.3)$ & $8(4.7)$ & $21(15.8)$ & \\
\hline Middle Atlantic & $1,193(17.2)$ & $221(16.8)$ & $588(17.5)$ & $211(15.6)$ & $57(18.2)$ & 55 (19.9) & $35(20.3)$ & $26(19.5)$ & \\
\hline South Atlantic & $1,371(19.8)$ & $291(22.1)$ & $655(19.5)$ & $241(17.8)$ & $50(15.9)$ & 76 (27.5) & $38(22.1)$ & $20(15.0)$ & \\
\hline $\begin{array}{l}\text { East North } \\
\text { Central }\end{array}$ & $1,372(19.8)$ & 235 (17.9) & 695 (20.7) & 283 (20.9) & $69(22.0)$ & $46(16.7)$ & $19(11.0)$ & 25 (18.8) & \\
\hline $\begin{array}{l}\text { East South } \\
\text { Central }\end{array}$ & 269 (3.9) & $47(3.6)$ & 125 (3.7) & $61(4.5)$ & $10(3.2)$ & $11(4.0)$ & $11(6.4)$ & $4(3.0)$ & \\
\hline $\begin{array}{l}\text { West North } \\
\text { Central }\end{array}$ & 595 (8.6) & $89(6.8)$ & $300(8.9)$ & 119 (8.8) & 35 (11.1) & $15(5.4)$ & $26(15.1)$ & $11(8.3)$ & \\
\hline $\begin{array}{l}\text { West South } \\
\text { Central }\end{array}$ & $311(4.5)$ & $55(4.2)$ & $144(4.3)$ & $62(4.6)$ & $17(5.4)$ & $14(5.1)$ & $15(8.7)$ & $4(3.0)$ & \\
\hline Mountain & 269 (3.9) & $54(4.1)$ & $136(4.0)$ & $47(3.5)$ & $8(2.5)$ & $11(4.0)$ & $8(4.7)$ & $5(3.8)$ & \\
\hline Pacific & $880(12.7)$ & $193(14.7)$ & 394 (11.7) & 177 (13.1) & $51(16.2)$ & 36 (13.0) & $12(7.0)$ & 17 (12.8) & \\
\hline
\end{tabular}

Values are in number (\%) unless otherwise indicated. IQR, interquartile range; AJCC, American Joint Committee on Cancer. 


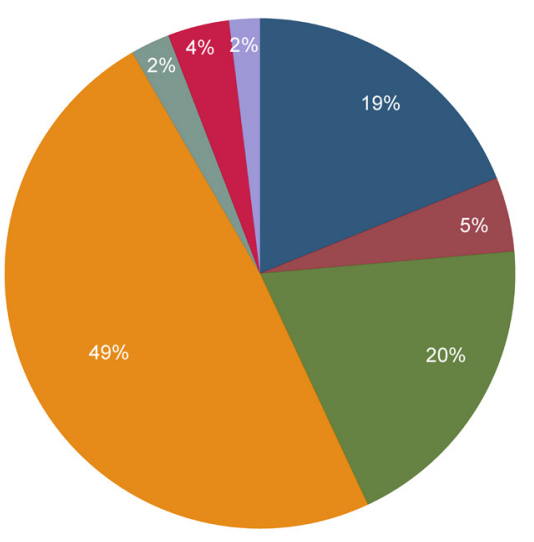

$\mathrm{N}=6,922$

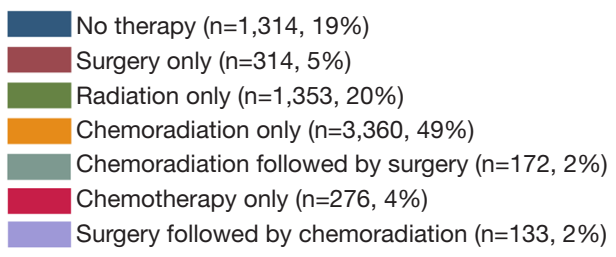

Figure 2 Pie chart of the treatment type of the cohort. Majority of the patients underwent chemoradiation only (49\%), radiation only (20\%) or no therapy (19\%).

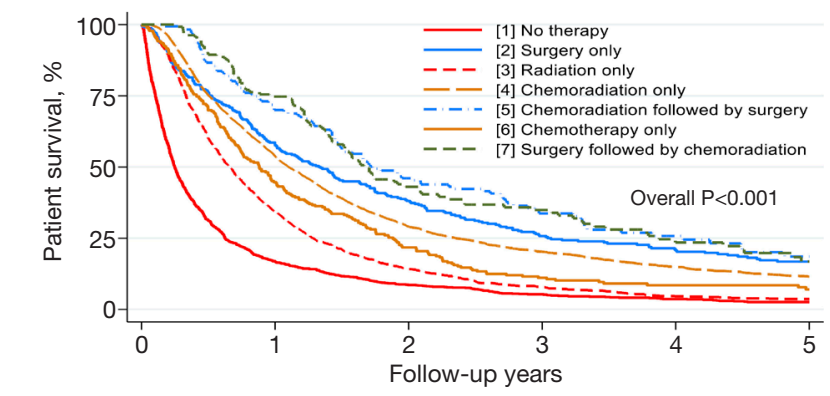

Number at risk

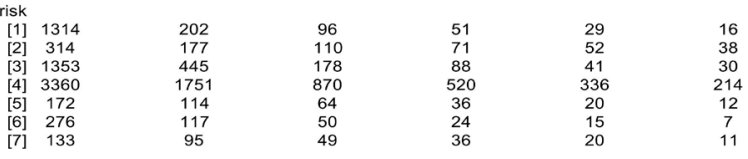

Figure 3 Kaplan Meier survival curve of the entire cohort. Patients who had treatment for esophageal cancer had significantly better survival than the patients who did not have treatment.

review of the literature of esophageal cancer outcomes in the elderly suggested that the 5-year overall survival range from $0 \%$ to $49 \%$ (13). Treatment-related complications are seen in up to $70 \%$ of patients, including chemotherapyrelated grade $\geq 3$ toxicity for $22-36 \%$ and surgeryrelated complications seen in $27-69 \%$ (13). Although the reported numbers are in disparity, numerous studies have
Table 2 Multivariable Cox proportional hazard model of risk for mortality for entire cohort

\begin{tabular}{lcc}
\hline \multirow{2}{*}{ Factor } & \multicolumn{2}{c}{5 years } \\
\cline { 2 - 3 } & $\begin{array}{c}\text { Adjusted HR } \\
(95 \% \mathrm{Cl})\end{array}$ & P value \\
\hline Treatment & (reference) & \\
No therapy & $0.34(0.29,0.39)$ & $<0.001$ \\
Surgery only & $0.56(0.51,0.60)$ & $<0.001$ \\
Radiation only & $0.35(0.33,0.38)$ & $<0.001$ \\
Chemoradiation only & $0.25(0.21,0.31)$ & $<0.001$ \\
Chemoradiation followed by & & \\
surgery & $0.45(0.39,0.52)$ & $<0.001$ \\
Chemotherapy only & $0.26(0.21,0.32)$ & $<0.001$ \\
Surgery followed by chemoradiation & $1.03(1.02,1.04)$ & $<0.001$ \\
Age at diagnosis & $1.07(1.01,1.14)$ & 0.02 \\
Male sex & & \\
Ethnicity & $($ reference $)$ & \\
Non-Hospanic & $0.93(0.79,1.10)$ & 0.38 \\
Hispanic & $1.15(1.04,1.28)$ & 0.01 \\
Unknown & &
\end{tabular}

Charlson-Deyo score

$\begin{array}{lcc}0 & \text { (reference) } & \\ 1 & 1.22(1.14,1.29) & <0.001 \\ 2 & 1.42(1.27,1.58) & <0.001 \\ \geq 3 & 1.17(0.99,1.38) & 0.06 \\ \text { Adenocarcinoma } & 0.91(0.86,0.98) & 0.01\end{array}$

AJCC clinical stage 3 (versus stage 2) $1.39(1.32,1.46)<0.001$

Facility type

Community cancer program

(reference)

Comprehensive community cancer $0.95(0.87,1.04) \quad 0.27$ program

Academic/research program $\quad 0.84(0.76,0.92)<0.001$

Integrated network cancer program $0.98(0.88,1.08) \quad 0.66$

C-statistic: 0.66. HR, hazard radio; $\mathrm{Cl}$, confidence interval; AJCC, American Joint Committee on Cancer.

unanimously indicated that the elderly with esophageal cancer have higher proportions of treatment morbidity, poorer disease-free outcomes, and shorter overall survival (14-16). However, it does not provide information regarding 
Table 3 Baseline characteristics of propensity matched group

\begin{tabular}{|c|c|c|c|c|c|c|c|c|}
\hline Characteristics & \multicolumn{4}{|c|}{ Before matching } & \multicolumn{4}{|c|}{ After matching } \\
\hline $\begin{array}{l}\text { Age at diagnosis, } \\
\text { median (IQR) }\end{array}$ & $83.0(81.0,85.0)$ & $81.0(80.0,82.5)$ & -0.76 & $<0.001$ & $82.0(80.0,83.0)$ & $82.0(81.0,83.0)$ & 0.03 & 0.86 \\
\hline White & 3,120 (92.9) & $163(94.8)$ & -0.08 & 0.34 & 77 (92.8) & $78(94.0)$ & -0.05 & 0.76 \\
\hline \multicolumn{9}{|l|}{ Ethnicity } \\
\hline Non-Hispanic & $3,069(91.3)$ & $164(95.3)$ & 0.16 & 0.07 & $80(96.4)$ & $79(95.2)$ & -0.06 & 0.70 \\
\hline Not insured & $9(0.3)$ & $1(0.6)$ & 0.05 & 0.45 & $0(0.0)$ & $0(0.0)$ & - & - \\
\hline $\begin{array}{l}\text { Private insurance/ } \\
\text { managed care }\end{array}$ & $263(7.8)$ & $15(8.7)$ & 0.03 & 0.67 & $8(9.6)$ & $6(7.2)$ & -0.09 & 0.58 \\
\hline Medicaid & $42(1.3)$ & $1(0.6)$ & -0.07 & 0.44 & $1(1.2)$ & $1(1.2)$ & 0.00 & 1.00 \\
\hline Medicare & 2,924 (87.0) & $150(87.2)$ & 0.01 & 0.94 & $71(85.5)$ & $74(89.2)$ & 0.11 & 0.49 \\
\hline Other government & $49(1.5)$ & $1(0.6)$ & -0.09 & 0.34 & $1(1.2)$ & $1(1.2)$ & 0.00 & -0.09 \\
\hline $\begin{array}{l}\text { Insurance status } \\
\text { unknown }\end{array}$ & $73(2.2)$ & $4(2.3)$ & 0.01 & 0.89 & $2(2.4)$ & $1(1.2)$ & 0.56 & 0.051 \\
\hline Urban & $3,195(95.1)$ & $159(92.4)$ & -0.11 & 0.01 & $77(92.8)$ & $76(91.6)$ & -0.05 & 0.77 \\
\hline \multicolumn{9}{|l|}{ Charlson-Deyo score } \\
\hline 0 & $2,437(72.5)$ & $119(69.2)$ & -0.07 & 0.34 & $58(69.9)$ & $59(71.1)$ & 0.03 & 0.87 \\
\hline 1 & $696(20.7)$ & $42(24.4)$ & 0.09 & 0.24 & $18(21.7)$ & $18(21.7)$ & 0.00 & 1.00 \\
\hline 2 & $161(4.8)$ & $10(5.8)$ & 0.05 & 0.54 & $6(7.2)$ & $5(6.0)$ & -0.05 & 0.76 \\
\hline$\geq 3$ & $66(2.0)$ & $1(0.6)$ & -0.12 & 0.20 & $1(1.2)$ & $1(1.2)$ & 0.00 & 1.00 \\
\hline \multicolumn{9}{|l|}{ Primary site } \\
\hline Cervical esophagus & $112(3.3)$ & $0(0.0)$ & -0.26 & 0.02 & $0(0.0)$ & $0(0.0)$ & - & - \\
\hline Thoracic esophagus & $103(3.1)$ & $3(1.7)$ & -0.09 & 0.32 & $0(0.0)$ & $1(1.2)$ & 0.16 & 0.32 \\
\hline $\begin{array}{l}\text { Abdominal } \\
\text { esophagus }\end{array}$ & $14(0.4)$ & $0(0.0)$ & -0.09 & 0.40 & $0(0.0)$ & $0(0.0)$ & - & - \\
\hline
\end{tabular}

Table 3 (continued) 
Table 3 (continued)

\begin{tabular}{|c|c|c|c|c|c|c|c|c|}
\hline Characteristics & \multicolumn{4}{|c|}{ Before matching } & \multicolumn{4}{|c|}{ After matching } \\
\hline Upper third & $201(6.0)$ & $6(3.5)$ & -0.12 & 0.17 & $2(2.4)$ & $3(3.6)$ & 0.07 & 0.65 \\
\hline Lower third & $2,051(61.0)$ & $132(76.7)$ & 0.34 & $<0.001$ & $52(62.7)$ & $62(74.7)$ & 0.26 & 0.09 \\
\hline Overlapping lesion & $152(4.5)$ & $10(5.8)$ & 0.06 & 0.43 & $8(9.6)$ & $5(6.0)$ & -0.13 & 0.39 \\
\hline Esophagus, NOS & $222(6.6)$ & $3(1.7)$ & -0.25 & 0.01 & $3(3.6)$ & $2(2.4)$ & -0.07 & 0.65 \\
\hline Adenocarcinoma & $2,137(63.6)$ & $141(82.0)$ & 0.42 & $<0.001$ & $59(71.1)$ & $64(77.1)$ & 0.14 & 0.38 \\
\hline \multicolumn{9}{|c|}{ AJCC clinical stage group } \\
\hline Stage 2 & $1,761(52.4)$ & $88(51.2)$ & -0.03 & 0.75 & $32(38.6)$ & $44(53.0)$ & 0.29 & 0.06 \\
\hline Stage 3 & $1,599(47.6)$ & $84(48.8)$ & 0.03 & 0.75 & $51(61.4)$ & $39(47.0)$ & -0.29 & 0.06 \\
\hline
\end{tabular}

IQR, interquartile range; AJCC, American Joint Committee on Cancer.

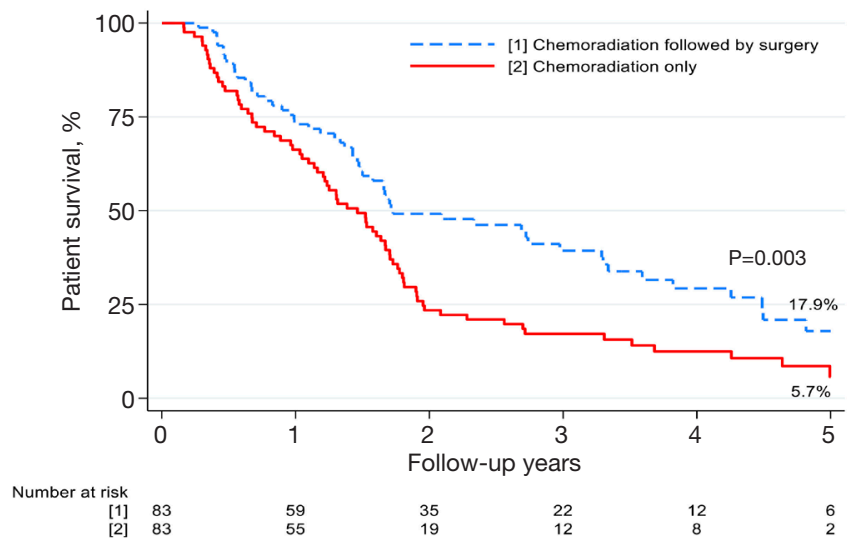

Figure 4 Kaplan Meier survival curve of the propensity matched groups. Patients who had chemoradiation followed by surgery had significantly better 5 -year survival than patients who did not have surgery $(17.9 \%$ vs. $5.7 \%, \mathrm{P}=0.003)$.
Table 4 Multivariable Cox proportional hazard model for risk for mortality in propensity matched group

\begin{tabular}{lll}
\hline Factor & Adjusted HR $(95 \% \mathrm{Cl})$ P value \\
\hline $\begin{array}{l}\text { Treatment } \\
\text { Chemoradiation only }\end{array}$ & (reference) & \\
$\begin{array}{l}\text { Chemoradiation followed by } \\
\text { surgery }\end{array}$ & $0.49(0.32,0.75)$ & 0.001 \\
Age at diagnosis & $1.13(1.03,1.24)$ & 0.01 \\
Male sex & $1.36(0.76,2.43)$ & 0.30 \\
Non-White race & $2.17(0.64,7.37)$ & 0.21 \\
Ethnicity & \\
Non-Hispanic & $($ reference $)$ & \\
Hispanic & $0.46(0.25,0.83)$ & 0.01 \\
$\quad$ Unknown & $1.27(0.63,2.57)$ & 0.51 \\
Urban & $1.22(0.63,2.36)$ & 0.57 \\
\hline
\end{tabular}

Table 4 (continued) 
Table 4 (continued)

\begin{tabular}{|c|c|c|}
\hline Factor & Adjusted HR (95\% Cl) & $P$ value \\
\hline \multicolumn{3}{|l|}{ Charlson-Deyo score } \\
\hline 0 & (reference) & \\
\hline 1 & $1.65(0.98,2.77)$ & 0.06 \\
\hline 2 & $1.40(0.81,2.42)$ & 0.23 \\
\hline$\geq 3$ & $0.54(0.06,5.23)$ & 0.59 \\
\hline \multicolumn{3}{|l|}{ Primary site } \\
\hline Upper and middle third & (reference) & \\
\hline Lower third & $1.14(0.73,1.76)$ & 0.57 \\
\hline Esophagus, NOS & $1.37(0.62,2.99)$ & 0.44 \\
\hline Adenoma/adenocarcinoma & $1.21(0.68,2.17)$ & 0.52 \\
\hline $\begin{array}{l}\text { Diagnostic and/or staging } \\
\text { procedure performed }\end{array}$ & $1.95(0.93,4.10)$ & 0.08 \\
\hline $\begin{array}{l}\text { AJCC clinical stage } 3 \text { (versus } \\
\text { stage 2) }\end{array}$ & $1.27(0.83,1.94)$ & 0.27 \\
\hline \multicolumn{3}{|l|}{ Facility type } \\
\hline Community cancer program & (reference) & \\
\hline $\begin{array}{l}\text { Comprehensive community } \\
\text { cancer program }\end{array}$ & $1.38(0.58,3.28)$ & 0.47 \\
\hline Academic/research program & $1.00(0.41,2.42)$ & 1.00 \\
\hline $\begin{array}{l}\text { Integrated network cancer } \\
\text { program }\end{array}$ & $0.81(0.31,2.09)$ & 0.66 \\
\hline \multicolumn{3}{|l|}{ Facility location } \\
\hline New England & (reference) & \\
\hline Middle Atlantic & $0.84(0.42,1.67)$ & 0.62 \\
\hline South Atlantic & $0.81(0.39,1.68)$ & 0.57 \\
\hline East North Central & $0.68(0.30,1.58)$ & 0.38 \\
\hline East South Central & $0.19(0.04,0.82)$ & 0.03 \\
\hline West North Central & $0.74(0.32,1.74)$ & 0.49 \\
\hline West South Central & $0.83(0.21,3.26)$ & 0.79 \\
\hline Mountain & $0.33(0.12,0.90)$ & 0.03 \\
\hline Pacific & $0.66(0.29,1.52)$ & 0.33 \\
\hline
\end{tabular}

C-statistic: 0.65 . Cox model was run with robust standard errors that account for the clustering in matched pairs. HR, hazard ratio; $\mathrm{Cl}$, confidence interval; AJCC, American Joint Committee on Cancer. the best therapy for this group of patients and most elderly patients often are not even considered for surgery due to perceived high risk (8). This finding is consistent with our data where a grand total of 1,314 patients did not receive any treatment.

In addressing the question whether the surgery should be considered in octogenarians with locally advanced esophageal cancer, we found that octogenarians had improved survival when surgery was incorporated as part of their treatment plan. Analysis of this cohort showed that therapy that included surgery such as surgery only, surgery followed by chemoradiation therapy, and chemoradiation therapy followed by surgery had the most improved survival compared to other modalities. In addition, chemoradiation only provided improved survival compared to monotherapy of chemotherapy only or radiation only. All these treatment paradigms provided more improved survival compared to no therapy. However, the treatment paradigm with surgery was rarely used, and most octogenarians with locally advanced esophageal cancer received definitive chemoradiation therapy only.

A possible reason for patients not undergoing surgery after chemoradiation may be that this group of patients had progression of the disease; thus, they were not offered an operation. However, the rate of patients who tend to progress to metastatic disease after chemoradiation in published studies is $4-10 \%(3,17)$. Thus, $48 \%$ of the patients not undergoing surgery in this cohort cannot be explained by disease progression alone. Other reasons for not undergoing surgical treatment after chemoradiation are significant decrease in performance status or death from chemoradiation. Moreover, a group of patients who decline surgery due to complete pathologic response (18). Lastly, these patients were not offered a surgery after their chemoradiation therapy may be because the providers thought that the surgery would not improve the patient's overall survival. The providers were likely to an assessment indicating that the patient might not fit for surgery. In our cohort, our multivariable analysis for mortality showed that in addition to no therapy, a higher Charlson-Deyo score was associated with an increased risk for mortality. As patients get older, they will have higher comorbidities and get to a point where cardiovascular disease mortality will exceed for cancer. Thus, the determinant of surgical candidacy lies within the impact of comorbidities in their overall mortality (19). Therefore, patients who are not surgical candidates due to their comorbidities should not undergo surgery. 
One of the limitations of evaluating the overall survival based solely on the therapy is that there is likely an introduction of bias due to the difference in the patients' characteristics. The patients who underwent chemoradiation therapy might not have been surgical candidates due to high comorbidities (20). To overcome this limitation, we performed a propensity score match for all the characteristics that contribute to mortality among patients who underwent the most common therapy of chemoradiation only to chemoradiation followed by surgery. Thus, matching those patients who had surgery to those patients who did not have surgery after chemoradiation therapy. In this balanced group, we found that chemoradiation followed by surgery was associated with significantly improved survival compared to chemoradiation only. In the multivariable analysis of this cohort, the strongest factors that protected octogenarians from the risk of mortality came from chemoradiation followed by surgery and younger age. Thus, after controlling for potential confounders that may affect the outcome, there is still an overall survival benefit for patients undergoing surgery after chemoradiation therapy. The number of patients in the matched cohort who were not offered surgery following chemoradiation therapy was low, likely because the patient had a progression of the disease, or the patient was not a surgical candidate. However, there may be a subset of patients whose providers do not believe that the patient will have a survival benefit from surgery (20). Our study provides data on this group of patients and suggests that after chemoradiation therapy, octogenarians who are surgical candidates should undergo surgery since the addition of surgery has a significant survival benefit.

Our study has several limitations. First, the retrospective analysis does not carry the weight of a randomized controlled trial. In addition, the national database does not provide granular details of patient characteristics such as performance status or complication after treatment. However, the study offers the largest cohort of octogenarians with clinical locally advanced esophageal cancer; thus, it provides some insight into the cohort of patients. This study does not address patient preference or patient's quality of life with different treatment modalities that may have contributed to not considering surgery as a mainstay of their therapy. Finally, we assumed that patients who underwent surgical resection were surgically fit patients. It is unknown if the patients who were matched were surgically fit since that information is not gathered in the NCDB database.

Overall, this study provides insight into a cohort of patients that we will continue to see in our surgical practice as the population continues to live longer and longer. While most patients only receive chemoradiation therapy, their overall survival is improved with the addition of surgery in the treatment modality. Select octogenarians with locally advanced esophageal cancer should be considered for surgery.

\section{Acknowledgments}

Funding: None.

\section{Footnote}

Reporting Checklist: The authors have completed the STROBE reporting checklist. Available at https://dx.doi. org/10.21037/jtd-21-928

Conflicts of Interest: All authors have completed the ICMJE uniform disclosure form (available at https://dx.doi. org/10.21037/jtd-21-928). MPK has consulted for Veran, Medtronic, AstraZeneca and Intuitive Surgical. EYC has consulted for Veran. MPK serves as an unpaid editorial board member of Fournal of Thoracic Disease from Sep 2020 to Aug 2022. The other authors have no conflicts of interest to declare.

Ethical Statement: The authors are accountable for all aspects of the work in ensuring that questions related to the accuracy or integrity of any part of the work are appropriately investigated and resolved. The study was conducted in accordance with the Declaration of. Helsinki (as revised in 2013). The institutional review board approval was waived for this study since we performed an analysis of national de-identified patient data and individual consent for this retrospective analysis was waived.

Open Access Statement: This is an Open Access article distributed in accordance with the Creative Commons Attribution-NonCommercial-NoDerivs 4.0 International License (CC BY-NC-ND 4.0), which permits the noncommercial replication and distribution of the article with the strict proviso that no changes or edits are made and the original work is properly cited (including links to both the formal publication through the relevant DOI and the license). See: https://creativecommons.org/licenses/by-nc-nd/4.0/. 


\section{References}

1. Cancer Stat Facts: Esophageal Cancer: National Cancer Institute. Available online: https://seer.cancer.gov/statfacts/ html/esoph.html\#survival

2. Zhang Y. Epidemiology of esophageal cancer. World J Gastroenterol 2013;19:5598-606.

3. Shapiro J, van Lanschot JJB, Hulshof MCCM, et al. Neoadjuvant chemoradiotherapy plus surgery versus surgery alone for oesophageal or junctional cancer (CROSS): long-term results of a randomised controlled trial. Lancet Oncol 2015;16:1090-8.

4. Allum WH, Stenning SP, Bancewicz J, et al. Long-term results of a randomized trial of surgery with or without preoperative chemotherapy in esophageal cancer. J Clin Oncol 2009;27:5062-7.

5. Ando N, Kato H, Igaki H, et al. A randomized trial comparing postoperative adjuvant chemotherapy with cisplatin and 5-fluorouracil versus preoperative chemotherapy for localized advanced squamous cell carcinoma of the thoracic esophagus (JCOG9907). Ann Surg Oncol 2012;19:68-74.

6. Zeng Y, Liang W, Liu J, et al. Esophageal cancer in elderly patients: a population-based study. J Thorac Dis 2018;10:448-57.

7. Markar SR, Karthikesalingam A, Thrumurthy S, et al. Systematic review and pooled analysis assessing the association between elderly age and outcome following surgical resection of esophageal malignancy. Dis Esophagus 2013;26:250-62.

8. Tapias LF, Muniappan A, Wright CD, et al. Short and long-term outcomes after esophagectomy for cancer in elderly patients. Ann Thorac Surg 2013;95:1741-8.

9. Depypere L, Thomas M, Moons J, et al. Analysis of patients scheduled for neoadjuvant therapy followed by surgery for esophageal cancer, who never made it to esophagectomy. World J Surg Oncol 2019;17:89.

10. Austin PC. Balance diagnostics for comparing the

Cite this article as: Del Calvo H, Nguyen DT, Chan EY, Chihara R, Graviss EA, Kim MP. Surgery provides improved overall survival in surgically fit octogenarians with esophageal cancer after chemoradiation therapy. J Thorac Dis 2021;13(10):5875-5886. doi: 10.21037/jtd-21-928 distribution of baseline covariates between treatment groups in propensity-score matched samples. Stat Med 2009;28:3083-107.

11. Bakhos CT, Salami AC, Kaiser LR, et al. Outcomes of octogenarians with esophageal cancer: an analysis of the National Cancer Database. Dis Esophagus 2019;32:1-8.

12. Miyata H, Yamasaki M, Makino T, et al. Clinical Outcome of Esophagectomy in Elderly Patients With and Without Neoadjuvant Therapy for Thoracic Esophageal Cancer. Ann Surg Oncol 2015;22 Suppl 3:S794-801.

13. Skorus UA, Kenig J. Outcome of esophageal cancer in the elderly - systematic review of the literature. Wideochir Inne Tech Maloinwazyjne 2017;12:341-9.

14. Vlacich G, Samson PP, Perkins SM, et al. Treatment utilization and outcomes in elderly patients with locally advanced esophageal carcinoma: a review of the National Cancer Database. Cancer Med 2017;6:2886-96.

15. Morita M, Egashira A, Yoshida R, et al. Esophagectomy in patients 80 years of age and older with carcinoma of the thoracic esophagus. J Gastroenterol 2008;43:345-51.

16. Internullo E, Moons J, Nafteux P, et al. Outcome after esophagectomy for cancer of the esophagus and GEJ in patients aged over 75 years. Eur J Cardiothorac Surg 2008;33:1096-104.

17. Anderegg MC, de Groof EJ, Gisbertz SS, et al. 18F-FDG PET-CT after Neoadjuvant Chemoradiotherapy in Esophageal Cancer Patients to Optimize Surgical Decision Making. PLoS One 2015;10:e0133690.

18. Noordman BJ, Wijnhoven BPL, Lagarde SM, et al. Neoadjuvant chemoradiotherapy plus surgery versus active surveillance for oesophageal cancer: a stepped-wedge cluster randomised trial. BMC Cancer 2018;18:142.

19. Rocco G, Weder W. Lung surgery in the elderly today. Lung Cancer 2013;80:115-9.

20. Molena D, Stem M, Blackford AL, et al. Esophageal Cancer Treatment Is Underutilized Among Elderly Patients in the USA. J Gastrointest Surg 2017;21:126-36. 